\title{
Performance of self-reported adherence to oral pre-exposure prophylaxis (PrEP) among HIV heterosexual serodiscordant couples in rural Uganda
}

\author{
FM Kibengo ${ }^{1}$, E Ruzagira ${ }^{1}$, UM Bahemuka ${ }^{1}$, D Katende ${ }^{1}$, A Abaasa ${ }^{1}$, B Barin $^{2}$, F Priddy $^{3 *}$, J Haberer $^{4}$, A Kampala $^{1}$
}

From AIDS Vaccine 2012

Boston, MA, USA. 9-12 September 2012

\section{Background}

Adherence is one of the main determinants of PrEP efficacy. Most PrEP studies applied subjective adherence measures, which often produce overestimates and problematic efficacy data interpretation; creating a need for more objective measures. This study examines selfreported adherence to oral PrEP compared to Medical Events Monitoring System (MEMS).

\section{Methods}

Seventy-two HIV-uninfected partners (50\% women) in Uganda were randomized to daily or intermittent (Monday, Friday and within 2 hours after sex, not exceeding 1 dose/day) oral emtricitabine/tenofovir or placebo in a 2:1:2:1 ratio for four months. Adherence was assessed monthly by MEMS and self-reported taken or missed doses by timeline follow-back calendar. MEMS data was adjusted for extra openings without pill removal and removal of multiple pills. Non-fixed days within intermittent regimen were classified as adherent/non-adherent based on self-reported sex by SMS. Adherence rates by taken/missed doses were compared to raw MEMS data using Spearman correlation.

\section{Results}

Treatment and placebo groups were combined since adherence rates were similar. Daily raw MEMS adherence rate was significantly higher than fixed Intermittent rate $(\mathrm{p}=0.04)$ and post-coital dosing rate $(\mathrm{p}<0.0001)$. Raw MEMS data for daily and fixed intermittent dosing, poorly correlated with self-reported taken doses $(r=0.14$, $\mathrm{p}=0.42$ and $\mathrm{r}=0.01, \mathrm{p}=0.94$, respectively) and missed doses $(r=0.30, p=0.08$ and $r=0.07, p=0.69$, respectively). Self-reported daily adherence had high sensitivity but only fair positive predictive value (PPV) and very poor specificity. Self-reported adherence to intermittent fixed dosing had fair sensitivity, PPV and negative predictive value (NPV), but poor specificity. Self-reported adherence to post-coital dosing had very good sensitivity and NPV but poor specificity.

\section{Conclusion}

Median adherence for daily and intermittent fixed PrEP was high by objective and subjective measures, but poorly correlated. Adherence to post-coital dosing was poor and likely overestimated by self-report (possibly reflecting technical challenges of SMS). Self-reported adherence measures were highly sensitive but poorly specific.

\section{Author details}

'Medical Research Council/Uganda Virus Research Institute, Entebbe, Uganda. ${ }^{2}$ The EMMES Corporation, New York, NY, USA. ${ }^{3}$ International AIDS Vaccine Initiative, New York, USA. ${ }^{4}$ Massachusetts General Hospital Center for Global Health, Boston, MA, USA.

Published: 13 September 2012

doi:10.1186/1742-4690-9-S2-P213

Cite this article as: Kibengo et al:: Performance of self-reported adherence to oral pre-exposure prophylaxis (PrEP) among HIV heterosexual serodiscordant couples in rural Uganda. Retrovirology 2012 9(Suppl 2):P213.

International AIDS Vaccine Initiative, New York, USA

Full list of author information is available at the end of the article

C 2012 Kibengo et al; licensee BioMed Central Ltd. This is an Open Access article distributed under the terms of the Creative Commons 